\title{
Temporal evolution of aftershock sequences for re-entry protocol development in seismically active mines
}

\author{
J.A. Vallejos Queen's University, Canada; University of Chile, Chile \\ S.D. McKinnon Queen's University, Canada
}

\begin{abstract}
The temporal evolution of aftershock sequences following rockbursts, large magnitude events and blasts is studied using the modified Omori's law $n(t)=K(c+t)^{-p}$ for several aftershock sequences in a number of mines in Ontario, Canada. The parameters $K, c$, and $p$ are estimated by maximum likelihood and considering only the time interval that satisfies power-law behaviour $(c=0)$. Guidelines for the use of this equation for re-entry protocol development are proposed and discussed. Using Monte Carlo simulation, isoprobability curves for representing the time decay of aftershock sequences are derived and used as a framework of reference for tracking in real time the seismic path of new sequences. Examples are presented to illustrate the application of the proposed methodology.
\end{abstract}

\section{Introduction}

The single most important characteristic of mining seismicity relevant to the development of re-entry protocols is that following large seismic events or blasts, there is a short-term increase in levels of seismic activity that over time decays to background levels. An example of this decay for the event rate parameter is presented in Figure 1 for a rockburst related sequence in a mine.
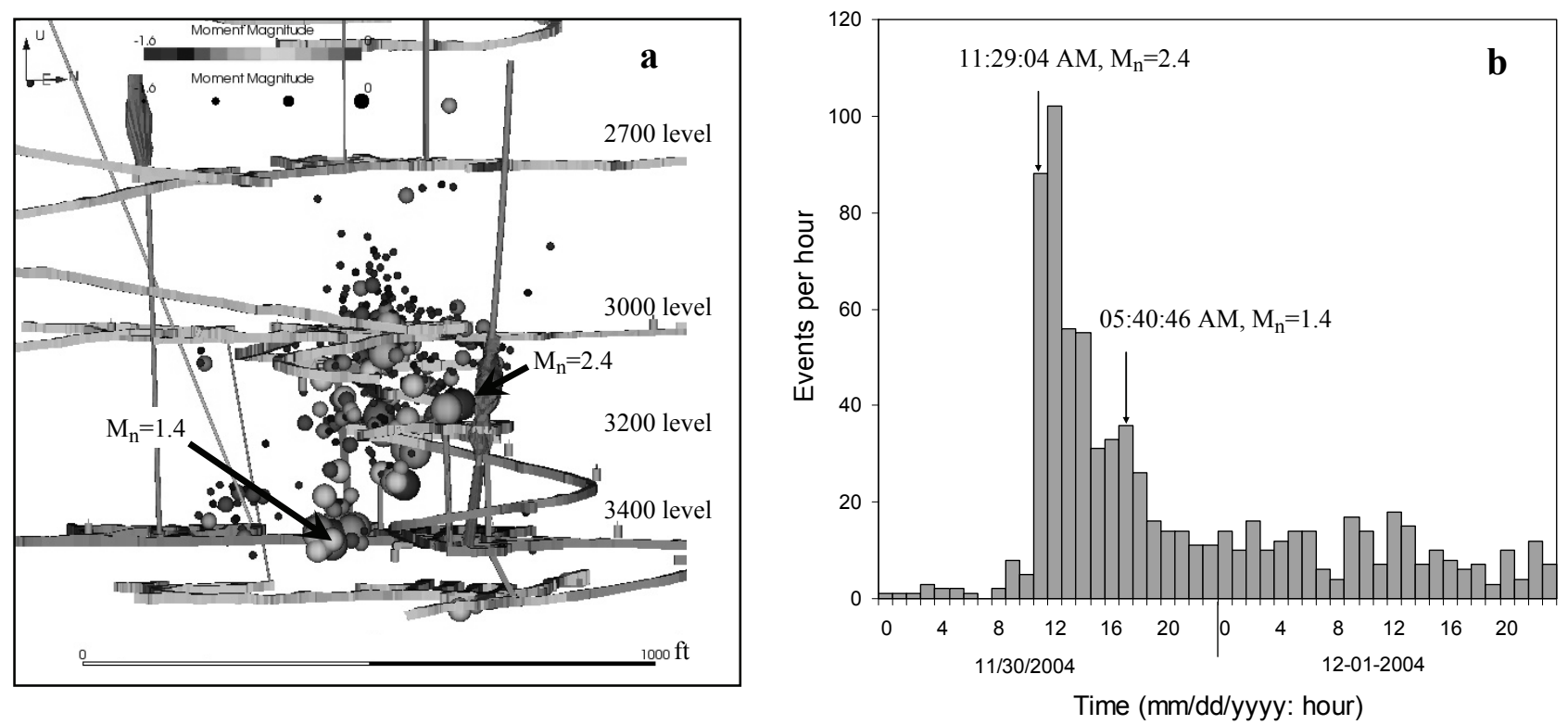

Figure 1 Example of increased levels of seismicity following a $M_{n}=2.4$ (Nuttli magnitude scale) rockburst at a mine: (a) spatial distribution of events in the affected zone; (b) events per hour as a function of the hour of the day. A second large magnitude event $\left(M_{n}=1.4\right)$ occurred in the same zone 6.2 hours after the initial event

Following the $\mathrm{M}_{\mathrm{n}}=2.4$ (Nuttli magnitude scale) shown in Figure 1, another large magnitude event $\left(M_{n}=1.4\right)$, occurred in the same zone approximately six hours after the initial event. In order to reduce the 
exposure of workers to this type of secondary occurrence, access to the affected areas is restricted for sufficient time to allow the decay of aftershock events. This is the main approach in re-entry strategies.

Immediately following large seismic events in mines, the frequency of events is high, but gradually decays to background levels over a matter of hours (see Figure 1b). This pattern follows the modified Omori's law (MOL) developed from observed decay rates of large earthquake aftershocks:

$$
n(t)=\frac{K}{(c+t)^{p}}
$$

Where:

$$
\begin{array}{lll}
n(t)= & \text { the rate of occurrence of aftershocks as a function of the time } t \text { elapsed since the main event. } \\
K & = & \text { the productivity parameter related to the total number of events in the sequence. } \\
c & = & \text { the offset time constant. } \\
p & = & \text { the parameter related to the speed of decay. }
\end{array}
$$

An important consequence of the MOL is the time dependent nature of rock strength (Scholz, 2003). The increase in aftershock activity in the source region is generally attributed to increases in stress and resulting damage to the rock fabric. This has been studied in various ways, including stress corrosion (Das and Scholz, 1981), and damage mechanics (Shcherbakov and Turcotte, 2004).

Given the significant number of mines using event rates for re-entry purposes (Vallejos and McKinnon, 2008); this paper studies the temporal evolution of event rate by using the modified Omori's law (Omori, 1894; Utsu, 1961). This law has been applied to several aftershock sequences in a number of mining operations in Ontario, Canada.

\section{$2 \quad$ Modified Omori's law in current re-entry practices}

Two specialised software packages are currently in use for re-entry purposes: (1) Mine Seismic Risk Analysis Program (MS-RAP), and (2) SeisWatch. The MS-RAP software, developed by the Australian Centre for Geomechanics (ACG), is not exclusively for re-entry analysis and includes several options for interpreting mining seismicity. The main tools for assessing re-entry are: (i) event rate decay, and (ii) cumulative energy release, considering the seismicity occurring within a defined spherical radius and time window around the main event coordinates (Heal et al., 2005). SeisWatch is an on-line seismic tool developed by the Engineering Seismology Group (ESG) as part of a research project at Creighton Mine (Van Dusen and Shumila, 2001). The purpose of this software is to monitor for a specified volume a range of seismic parameters including: seismic work, spatial clustering, strain rate, event rate decay-Omori analysis, and depth-event frequency histogram. Details of these parameters can be found in Malek and Leslie (2006). In this paper, the discussion will concentrate on the current application of the event rate decay-Omori analysis of these packages for re-entry protocol development (Figure 2). 

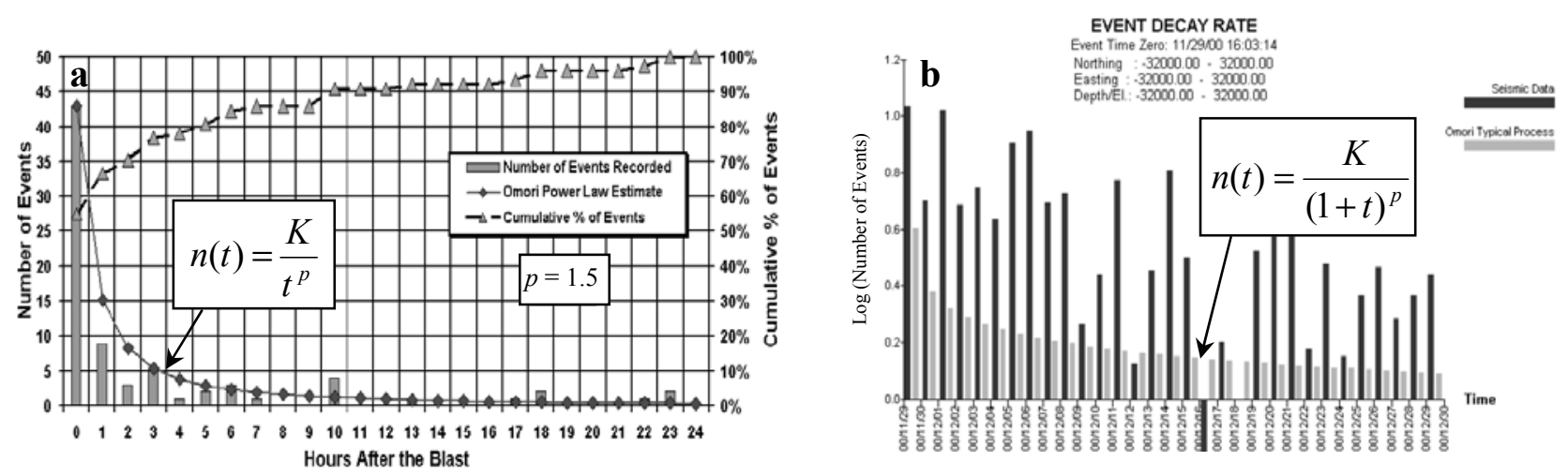

Figure 2 Event rate decay-Omori analysis available from two software packages currently in use for re-entry purposes following blasts or large magnitude events in mines: (a) MS-RAP (Hudyma et al., 2003); (b) SeisWatch (2007)

In both packages the event rate is displayed as a bar chart showing the number of events per time bin (one hour and one day in the MS-RAP and SeisWatch softwares respectively) following the initial event. Note also that SeisWatch employs a logarithmic scale on the vertical axis (Figure 2b).

As a reference of how the event rate decay can be expected to behave, both packages include a version of the MOL given by:

$$
\begin{gathered}
n(t)=\frac{K}{t^{p}} \text { MS-RAP } \\
n(t)=\frac{K}{(1+t)^{p}} \text { SeisWatch }
\end{gathered}
$$

With reference to the MS-RAP approach (Figure 2a), the parameter $c$ of Equation (1) is set equal to zero. Hudyma et al. (2003) mention that by back-analysis useful values for $K$ and $p$ can be determined; however no methodology to statistically determine these parameters was described. It can be observed in Figure 2a, that the number of events occurring during the first hour $N_{1}$ is coincident with the first point of Equation (2). Imposing $t=1$ in Equation (2) implies that $K=N_{1}$. A $p$ value equal to 1.5 is also indicated in Figure 2a, which based on the author's visual assessment gives a good fit to the decrease in time of the number of events. Hudyma et al. (2003), Heal et al. (2005) and Potvin (2008) indicated that a management decision is needed to decide what proportion of the seismic decay should occur prior to re-entry. Their analysis makes use of the cumulative number of events normalised by the corresponding total within a defined time period (secondary vertical axis in Figure 2a). Re-entry is defined at the time when $90 \%$ of the total events have occurred. The same rule was extended to seismic energy. The limitations of this method are that it can only be used for back-analysis (not for real time re-entry decision making), the result depends on the defined time period for the analysis, the $90 \%$ re-entry rule is arbitrary, and by definition, events or blasts that do not produce well-defined aftershock decay sequences can lead to long re-entry times.

Referring to SeisWatch (Figure $2 b$ ) the offset time constant $(c)$ is set equal to one. The parameters $K$ and $p$ are supposed to be user-supplied constants determined from a previously measured large magnitude event or rockburst. These parameters are fixed for future occurrences. It is well known from the crustal literature that the parameters $K$ and $p$ differ from sequence to sequence (see for example: Reasenberg and Jones, 1989; Utsu et al., 1995), and therefore fixing unique reference parameters based on a single past occurrence may not be completely representative of new aftershock sequences. In addition, if no large magnitude events/rockbursts are present in the database the equation cannot be calibrated.

Note that if time bins are used to represent the data and fit the MOL the estimate of the parameters will depend on the length of the selected time bin. Therefore, a uniform method that does not depend on time bins needs to be used for estimating the MOL parameters. This is crucial for developing guidelines of how the 
MOL can be quantitatively used in real-time for re-entry protocol development. These topics are reviewed and addressed in-depth later in this paper.

\section{$3 \quad$ Methodology}

\subsection{Identification of aftershock sequences}

There is no standard definition of aftershocks but several have been used for specific purposes (Isacks et al., 1967; Shlien and Toksoz, 1974; Kagan and Knopoff, 1976; Reasenberg, 1985; Ogata, 1988; Davis and Frohlich, 1991a, 1991b; Kagan and Jackson, 2006). Molchan and Dmitrieva (1992) suggest that aftershock identification depends on the research goals. The target of this research is to reflect current re-entry practices where the seismic data is used for re-entry decision-making within a volume of interest surrounding the blast or event region. Therefore, the analysis is relaxed to the temporal identification of aftershock sequences for the zone of interest without any formal consideration of the spatial distribution. For a specified zone, cluster or target volume, aftershock sequences are identified in time by using the identification provided by the mine personnel. When this identification is not available or reliable, the start of a sequence is identified by the ratios method (Frohlich and Davis, 1985). This method evaluates the ratio:

$$
r_{N_{B}-N_{A}}=\frac{T_{N_{A}}}{T_{N_{B}}}
$$

Where:

$T_{N_{A}}, T_{N_{B}}=$ origin times of the $N_{A}{ }^{\text {th }}$ and $N_{B}{ }^{\text {th }}$ events following and preceding the principal event respectively.

Equation (4) is continuously evaluated and the beginning of the sequence is identified if $r_{N_{B}-N_{A}}$ is smaller than a critical value generated by a Poisson process with a certain probability. For the analysis $N_{A}=1$, $N_{B}=5$ with a probability of $1 \%$ were used, giving a critical value of $r_{5-1}^{c}=0.002$ (Frohlich and Davis, 1985). The start of the sequence is defined if $r_{5-1}<=r_{5-1}^{c}$ for a group of at least three consecutive events. The first event of the group is identified as the principal event of the sequence. Events between principal events are considered as aftershocks. Note that this scheme does not attempt to give a formal classification of foreshocks/aftershocks, and that there is no restriction in the magnitude of the events in the sequence to be less than the principal event. Figure 3 presents an example of this approach for an active stoping area in a mine.

\subsection{Magnitude filtering}

A cut-off moment magnitude $M_{w}^{c}$ filter, selected at the magnitude bin with the highest frequency of events in a non-cumulative frequency-magnitude distribution is applied to the identified aftershock sequences (Figure 3e). The effect of this filter is to provide some degree of uniformity to the data as all events below this magnitude level may not be fully recorded by the microseismic monitoring system. Only seismic sequences with at least 10 events and two hours of duration after filtering are retained for the analysis.

\subsection{Estimate of the modified Omori's law parameters}

For each aftershock sequence the parameters $K, p$ and $c$ with their uncertainties are estimated by the method of maximum likelihood (Ogata, 1983). Given the occurrence times $t_{i}(i=1, \ldots, N)$ of the individual $N$ events in a time interval $\left[T_{A}, T_{B}\right]$ the log-likelihood function of Equation (1) can be written as:

$$
\ln L\left(K, p, c, T_{A}, T_{B}\right)=N \ln K-p \sum_{i=1}^{N} \ln \left(t_{i}+c\right)-K A\left(p, c, T_{A}, T_{B}\right)
$$

With: 


$$
A\left(p, c, T_{A}, T_{B}\right)= \begin{cases}\ln \left(T_{B}+c\right)-\ln \left(T_{A}+c\right) & \text { for } p=1 \\ {\left[\left(T_{B}+c\right)^{1-p}-\left(T_{A}+c\right)^{1-p}\right] /(1-p)} & \text { for } p \neq 1\end{cases}
$$

The maximum likelihood estimates (MLE) of the parameters $K, p$ and $c$ are those that maximise Equation (5). To evaluate whether the MOL fitted parameters adequately describe the time sequence, the Anderson-Darling statistic- $W^{2}$ (Anderson and Darling, 1954) is used:

$$
W^{2}=-N-\sum_{i=1}^{N} \frac{(2 i-1)}{N}\left[\ln \left(u_{i}\right)+\ln \left(1-u_{N+1-i}\right)\right]
$$

Where $u_{i}$ is the MOL cumulative density function for event $i$ :

$$
u_{i}= \begin{cases}\frac{\ln \left(t_{i}+c\right)-\ln \left(T_{A}+c\right)}{\ln \left(T_{B}+c\right)-\ln \left(T_{A}+c\right)} & \text { for } p=1 \\ \frac{\left(t_{i}+c\right)^{1-p}-\left(T_{A}+c\right)^{1-p}}{\left(T_{B}+c\right)^{1-p}-\left(T_{A}+c\right)^{1-p}} & \text { for } p \neq 1\end{cases}
$$

$W^{2}=0$ indicates a perfect fit to the data. Sequences with $W^{2} \leq 2$ have been previously considered to follow the MOL distribution, and those with $W^{2} \leq 1$ are considered to fit well (Nyffenegger, 1998; Nyffenegger and Frohlich, 1998, 2000).

The following steps are used to estimate the MOL parameters for a given seismic sequence:

1. Specify a target time interval $\left[T_{A}, T_{B}\right]$ within the seismic sequence.

2. Maximise the log-likelihood function (Equation (5)).

3. Test if the MOL fitted parameters adequately describe the time sequence with $W^{2}$ (Equation (7)).

In fact, there are numerous time intervals that satisfy $W^{2} \leq 1.0$ for a given seismic sequence. Vallejos and McKinnon (2010) determined that the MLE of the MOL parameter is sensitive to the time interval $\left[T_{A}, T_{B}\right]$ used to fit the equation. They recommended that for estimating consistent decay parameters it is necessary to consider the maximum time interval that satisfies a power-law MOL behaviour: $W^{2} \leq 1.0$ and $c=0$. They also proposed a uniform method for estimating the time interval of power-law decay, which is used in this paper. Some advantages of using the power-law MOL are: (i) the number of parameters that need to be set apriori for forecasting the decay is reduced to $K$ and $p$, (ii) the positive correlation between the parameters $c$ and $p$ (Gasperini and Lolli, 2006; Vallejos, 2010) is circumvented, and (iii) in terms of the physics of the problem, it is possible to have an estimate of the time at which the sequence actually starts the steady decay process $T_{S}$. 
$\mathbf{a}$
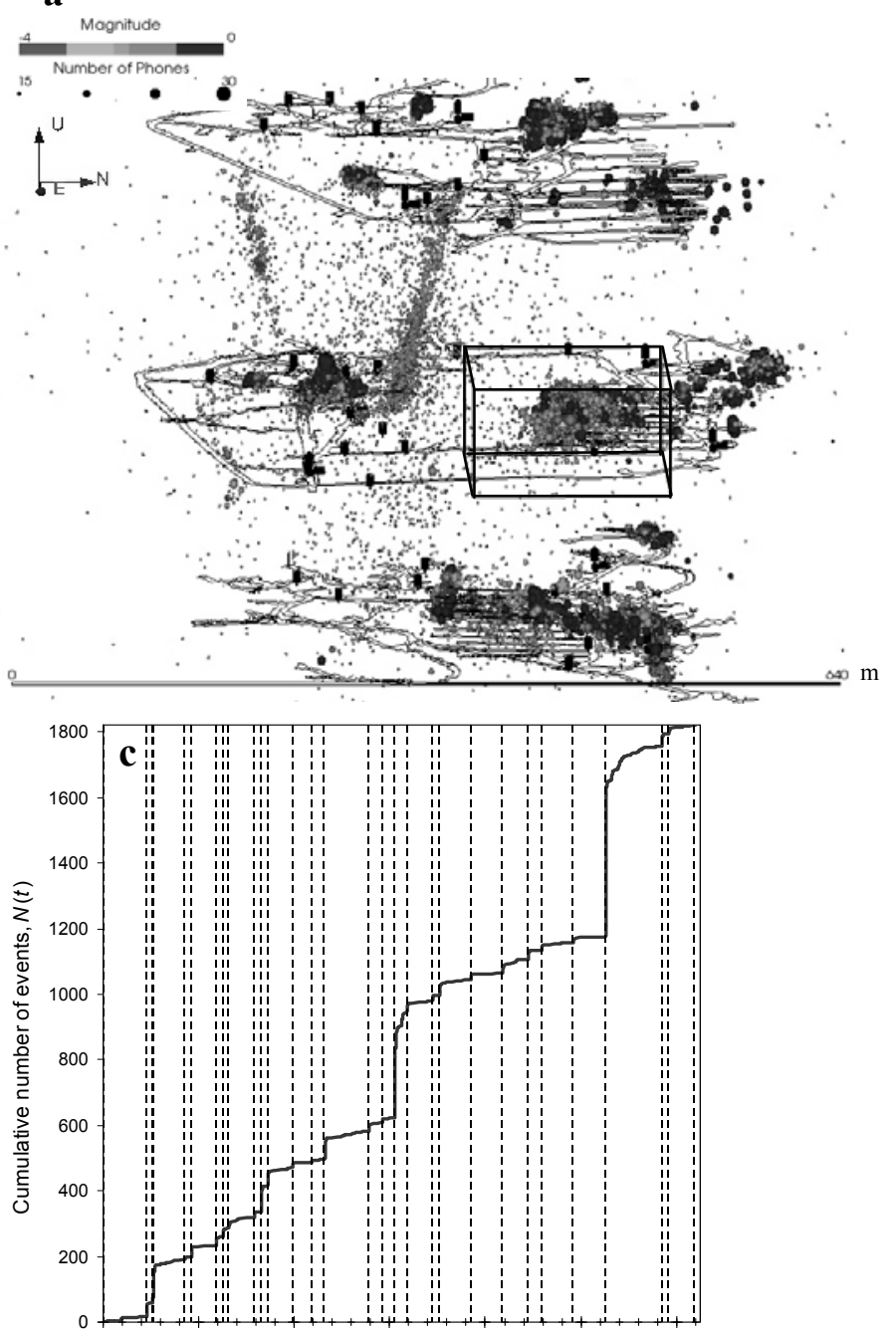
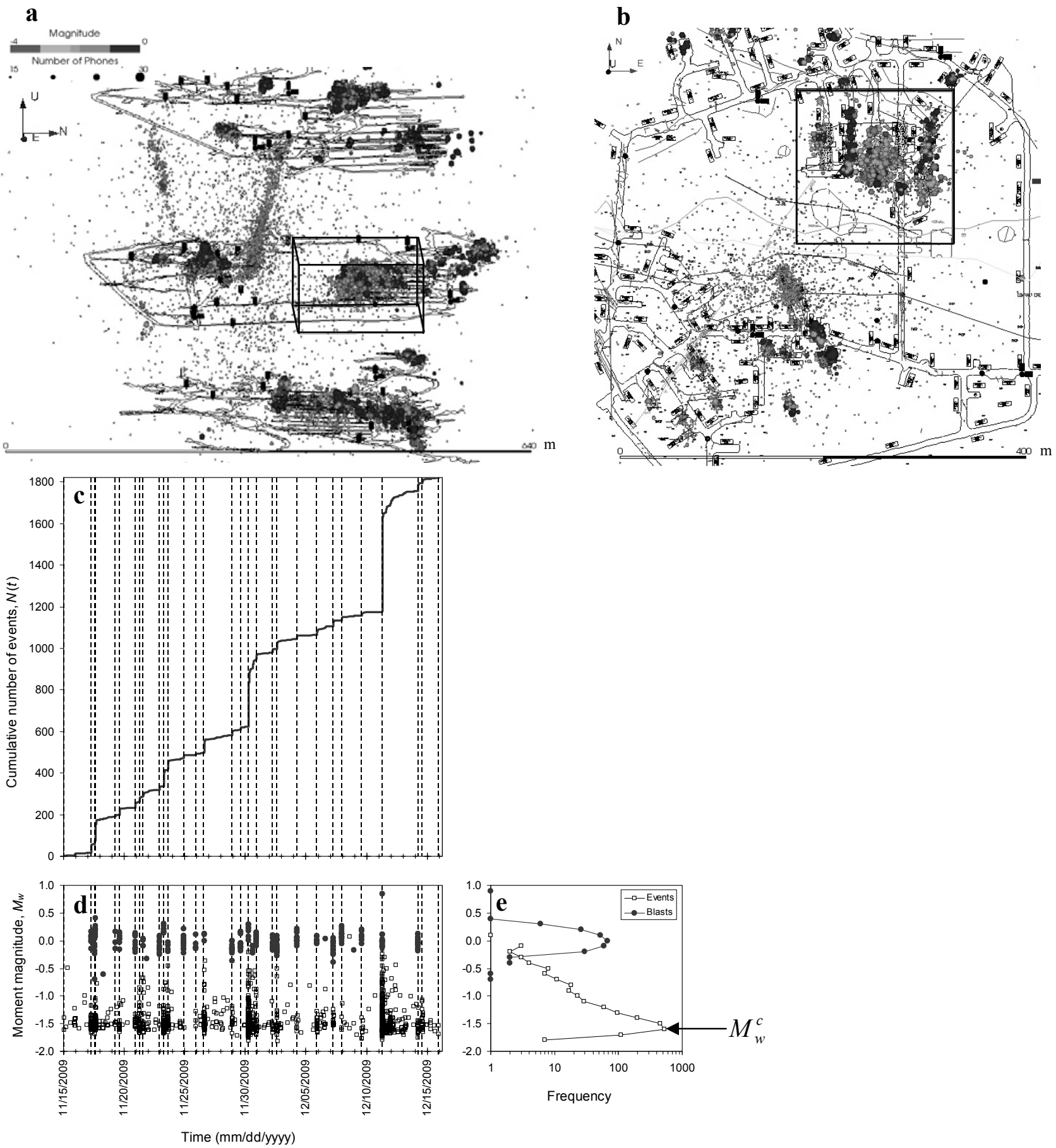

Figure 3 Aftershock sequence identification provided by the ratios method for an active stoping area in a mine for a one month time period. 3D (frame a) and plan (frame b) views of the stoping area. The study volume is outlined: (c) cumulative number of events-time for the selected volume; (d) magnitude-time plot; (e) non-cumulative frequency moment magnitude distribution. Vertical dashed lines represent the principal events identified by applying the ratios method. Events labelled as blasts are identified by full circles

\section{Data}

A total of 340 seismic sequences from nine different mining environments in Ontario, Canada (Table 1), are analysed using the methodology previously described. This wide variety of mining, geology and seismic settings is selected to test the applicability of the methodology and to evaluate the range of aftershock 
statistics that can be found in mining operations in Ontario. A brief description of mining methods, geology and microseismic monitoring systems can be found elsewhere (Vallejos and McKinnon, 2009; Vallejos and McKinnon, 2010; Vallejos, 2010).

Table 1 Number of seismic sequences retained for the analysis at each mining site

\begin{tabular}{lll}
\hline Mining Site & $\begin{array}{l}\text { Number of } \\
\text { Sequences }\end{array}$ & $M_{w}^{c}$ \\
\hline A & 28 & -2.0 \\
Copper Cliff North - 100/900OB & 51 & -1.6 \\
Craig - Zones 10/11 & 5 & -1.6 \\
Creighton - Deep & 44 & -1.5 \\
Kidd Creek - D mine & 70 & -1.9 \\
Macassa - 5036 longhole stopes & 49 & -1.2 \\
McCreedy East - 4400 Level 153OB & 24 & -2.1 \\
Nickel Rim South - Stopes 1480 Level & 46 & -1.6 \\
Williams - 4400 Level 153 OB & 23 & -1.9 \\
Total & 340 & \\
\hline
\end{tabular}

\section{$5 \quad$ Parameter statistics}

In this section the statistical results and interpretation of the start time of power-law decay $T_{S}$ and the power-law MOL parameters $p$ and $K$ are presented. The objectives are: (i) identify characteristic ranges and the probability distributions for representing the population of the power-law MOL parameters, and (ii) prepare the data for the Monte Carlo simulations.

\subsection{Start time of power-law decay}

Figure 4a presents the cumulative ascending distributions of start times of power-law decay $T_{S}$ for all the sequences analysed. It is seen that in $98 \%$ of the cases, the actual decay starts within one hour after the initial event, indicating that mining seismicity, aftershock sequences display non-power law behaviour only for short times $(<1$ hour). Several factors may be affecting the decay rate before the onset of the power-law decay, such as: overlapping of seismic records that make it difficult to identify and locate many events (Kagan, 2004), a complex process which the MOL equation is not able to adequately describe, or the sequence may actually begin gradually and build to a higher rate before the onset of smooth decay. This is likely to be due to undetected events missing from the early part of the sequence (Gross and Kisslinger, 1994).

\section{$5.2 p$ value}

The cumulative ascending distributions of $p$ values estimated for the time interval of power-law decay are presented in Figure $4 \mathrm{~b}$. The $p$ value varies from sequence to sequence, with most (95\%) being within a typical range of 0.4 to 1.6 , and mean values from 0.7 to 1.2 (Figure $4 \mathrm{~b}$ ). The most consistent statistical distribution for representing the population of $p$ values is determined by a log-normal distribution as indicated by the high observed significance levels of the Chi-Square test $\left(P_{C h i-S q}\right)$ in Figure $4 \mathrm{~b}$.

The variability of the $p$ value has been previously related to the fault system heterogeneity, surrounding lithosphere, local stress fields and crustal temperature (e.g. Mogi, 1967; Kisslinger and Jones, 1991; Kisslinger, 1996). 

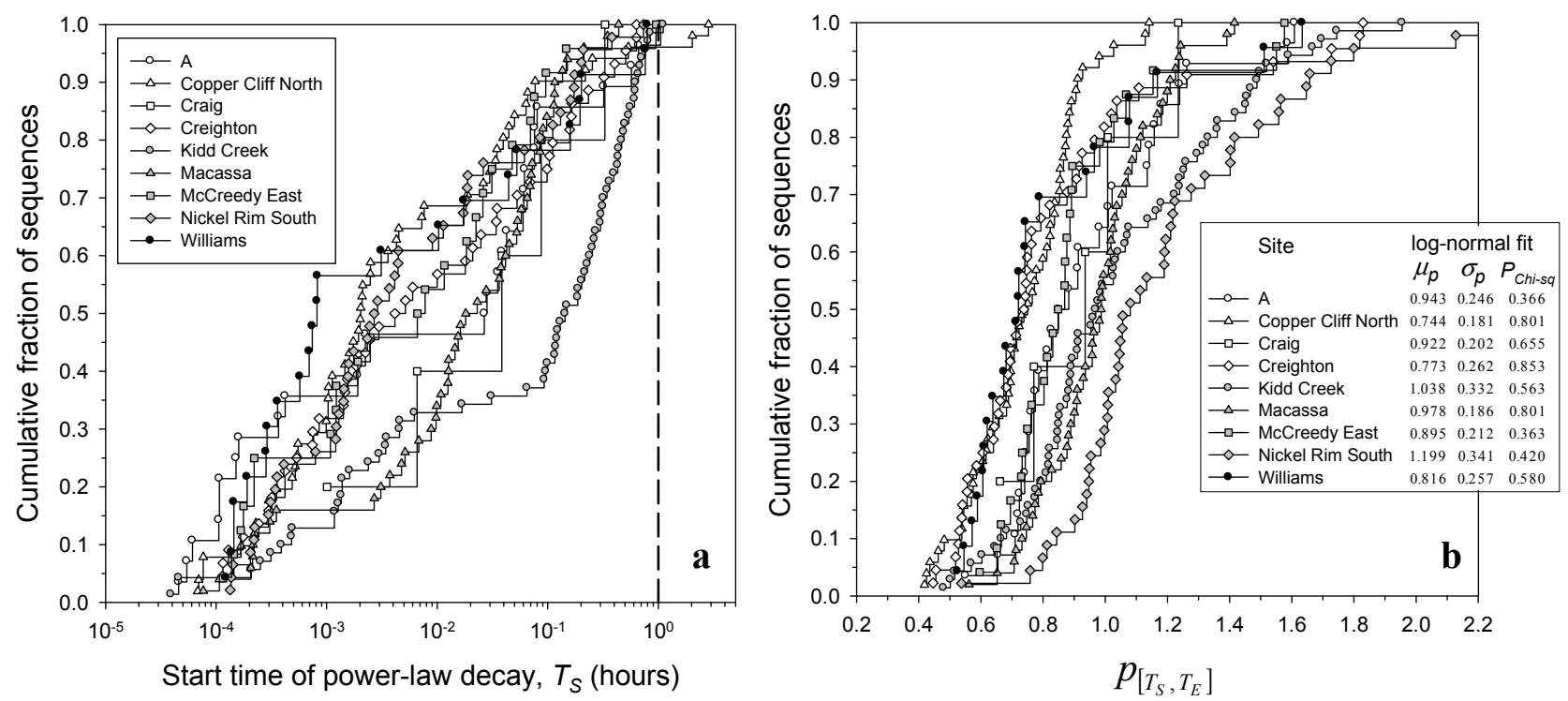

Figure 4 Cumulative ascending distributions for: (a) Start time of power-law decay $T_{S}$; (b) $p$ values estimated for the time interval of power-law decay $\left[T_{S}, T_{E}\right]$

\subsection{K value}

By definition for a sequence conforming to power-law decay, the parameter $K$ corresponds to the rate of activity one unit time after the principal event. Vallejos and McKinnon $(2009,2010)$ found that $K$ can be satisfactorily expressed by: $K=\kappa N_{1}$, where $N_{1}$ is the measured number of events occurring during the first hour after the principal event and $\kappa$ is a productivity ratio with a typical range between 0.25 and 0.50 , and an average of $0.35 \pm 0.07$. With this result and assuming the mean $p$ value of the site, it is possible to delineate the expected event decay rate one hour after the initial event. Although this guideline is extremely useful, the estimates of $\kappa$ were obtained by a least squares fit between $K$ and $N_{1}$, and some sequences with high $\kappa$ values were excluded from the analysis. In this paper, those previously excluded cases are included by representing the entire distribution of the $K$ values with a log-normal distribution (Figure 5). The parameters $\mu_{K}$ and $\sigma_{K}$ reflect the mean and dispersion of the activity rate of the aftershock sequences at each site. 


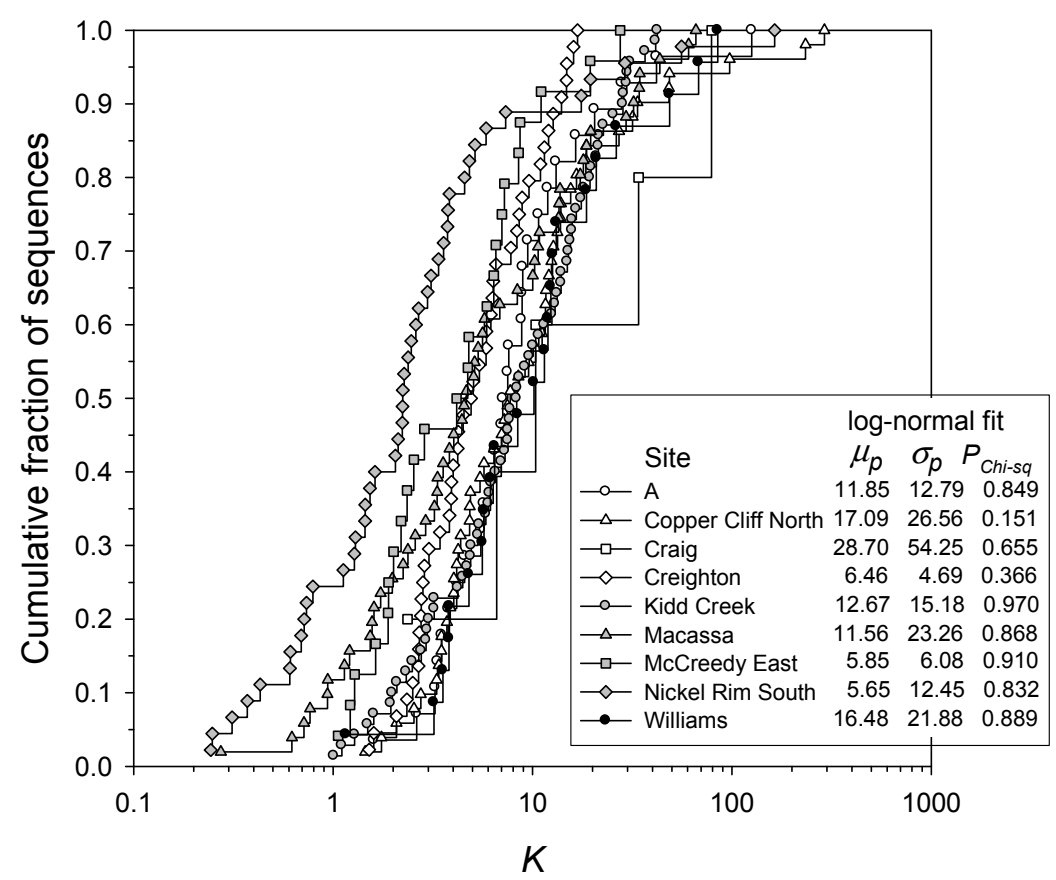

Figure 5 Cumulative ascending distributions for the $K$ values

\section{Re-entry protocol development}

\subsection{Time of maximum curvature of the MOL}

By tracing in time the curvature of the MOL a characteristic point emerges at the maximum curvature:

$$
T_{M C}=\left[K p \sqrt{\frac{2 p+1}{p+2}}\right]^{\frac{1}{p+1}}-c
$$

$T_{M C}$ has a physical attribute of a time sequence obeying a MOL decay suitable for use in re-entry protocols. It defines the transition between the highest to lowest event rate change (Vallejos and McKinnon, 2010).

\subsection{Monte Carlo simulations}

Instead of setting a unique or standard MOL curve as a reference against which to evaluate the sequence being evaluated for re-entry, a series of MOL curves reflecting both the variability and average conditions of the aftershock sequences of the site are introduced. At each time $t$ after a principal event, a Monte Carlo analysis is performed by using the well-defined distributions of the decay-law parameters $p$ and $K$ presented in Figure $4 \mathrm{~b}$ and Figure 5 respectively, on the cumulative version of the power-law MOL:

$$
N(t)=\int_{1}^{t} n(t) d t=\frac{K}{1-p}\left(t^{1-p}-1\right)
$$

This version of the MOL was preferred to avoid the use of time bins for representing the data. The curves obtained from Equation (10) are valid one hour after the principal event so they must be shifted to match the measured number of events occurring during the first hour. By establishing these curves one hour after the main event there is a $98 \%$ confidence level that the power-law decay has been established (Section 5.1). The resulting isoprobability curves (Figure 6a) are interpreted directly as the probability for the cumulative number of events to be lower than a certain value at a certain time after the main event and are referred to as the seismic envelopes. 
In addition to the seismic envelopes the time of maximum curvature $T_{M C}$ (Equation (9)), and the event rate at $T_{M C}$ are also incorporated into a Monte Carlo simulation with the distributions of $p$ and $K$. This simulations results in a maximum curvature boundary in the event rate diagram that is used to estimate $T_{M C}$ in real time, i.e. when the event rate crosses this boundary the maximum curvature has been reached (Figure 6b).
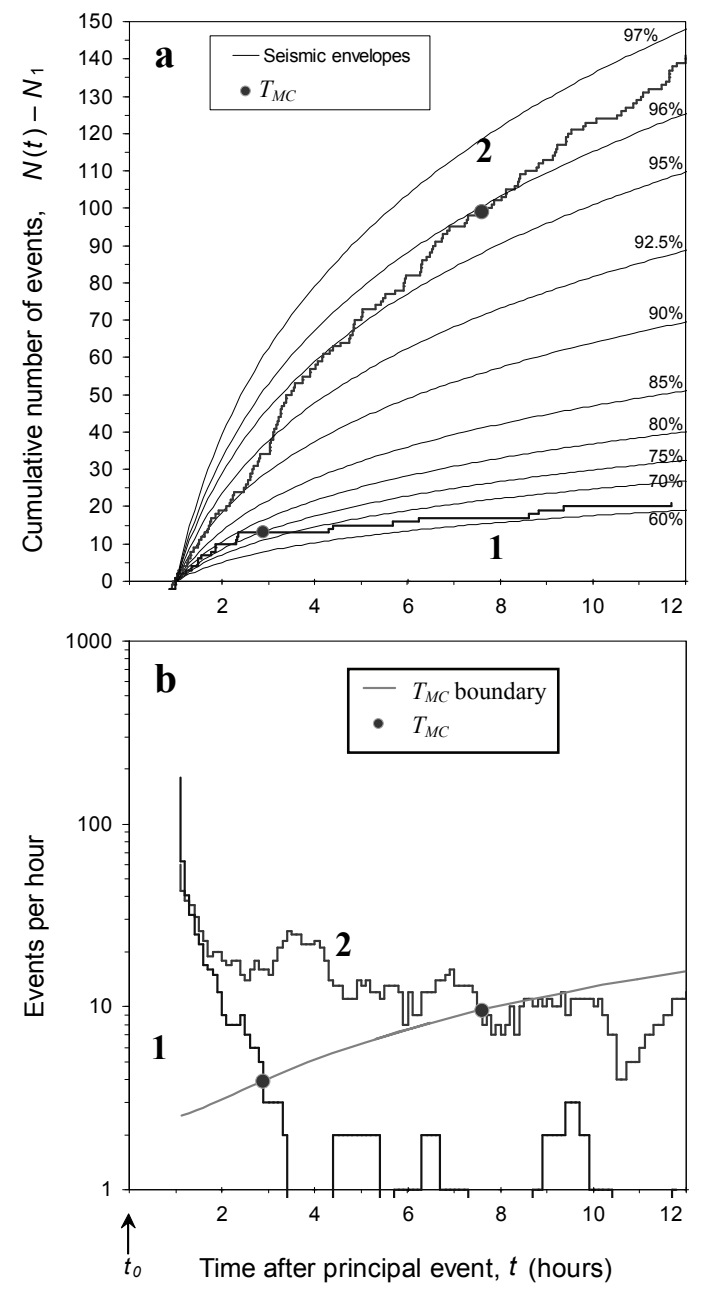

Figure 6 Illustration of the seismic envelopes (frame a) and event rate diagram (frame b) for two seismic paths. The cumulative number of events (frame a) were subtracted from the number of events occurring during the first hour in order to present both seismic paths in the same frame

The practical implications of the seismic envelopes (Figure 6a) are in order:

- They allow a classification to be made of the type of aftershock sequence under analysis based on the envelope being followed, i.e. it can be seen and tested in real-time to which level of probability the actual seismicity corresponds.

- Different speeds of decay can be identified by comparing the seismic path with the seismic envelopes.

- Speeds of decay higher than the average (path 1 in Figure 6a) are identified by a seismic path that terminates in a fast manner, crossing the seismic envelopes in a sub-horizontal direction. This type of behaviour indicates a sequence where most of the seismicity is associated with the main event. 
- Speeds of decay lower than the average (path 2 in Figure 6a) are indicated by a seismic path crossing several envelopes in a sub-vertical direction. This condition may arise mainly from two reasons: (i) the principal event had little influence and the new data is indistinguishable from background levels of seismicity and the seismic path crosses low percentile envelopes, or (ii) significant time clustering is occurring at long delay times after the main event suggesting a delayed ground response. The type of situation is clarified by comparing the actual rate of the sequence with a previously defined background level. This is to be incorporated into the event rate diagram (Figure 6b) and is addressed in the next section by the use of diurnal charts.

It is still not clear which factors are the most significant in controlling the speed of decay of mining-induced aftershock sequences. Using a rate-state dependent fault strength model, Dieterich (1994) indicated that $p>1$ may arise when the stresses on the fault surface decrease with time following the principal event. Similarly, laboratory experiments suggest that the $p$ value decreases with increasing load level (Lockner, 1993; Rudajev et al., 1996). Assuming that these statements are correct, then determining real-time $p$ values can be particularly useful for re-entry protocol development. However, this has been proven to be sensitive (Vallejos and McKinnon, 2010). It is considered here that a better understanding of the behaviour of aftershock sequences is obtained if the actual sequence is compared with the statistical analysis of previous occurrences. This approach assumes that the seismic environment responds in a particular manner to the mining process and can be represented by statistical parameters.

\subsection{Background levels-Diurnal charts}

As a simple and practical statistical tool, the use of diurnal charts is recommended for estimating background levels for re-entry protocol development (Figure 7a). This tool is based on the superposition of seismicity in a 24-hour chart which could include several months of observations at a particular location. In the example presented in Figure 7a, it is clear that there is a typical background level of seismic activity plus two daily blasting shifts, each of which triggers an increase in seismicity that decays over a three to four hour time period. Visual inspection of Figure 7a suggests that the hours of highest activity are: 4, 5, 6, 7, 16, 17, 18 and 19 , yielding a total percentage of background activity of $A_{T \%}=42.2 \%$. The background level $B$ is then evaluated by: $B=A_{T \%} A / T$, where $A$ is the total activity for the time period of interest and $T$ is the total non-omitted time bins of activity.
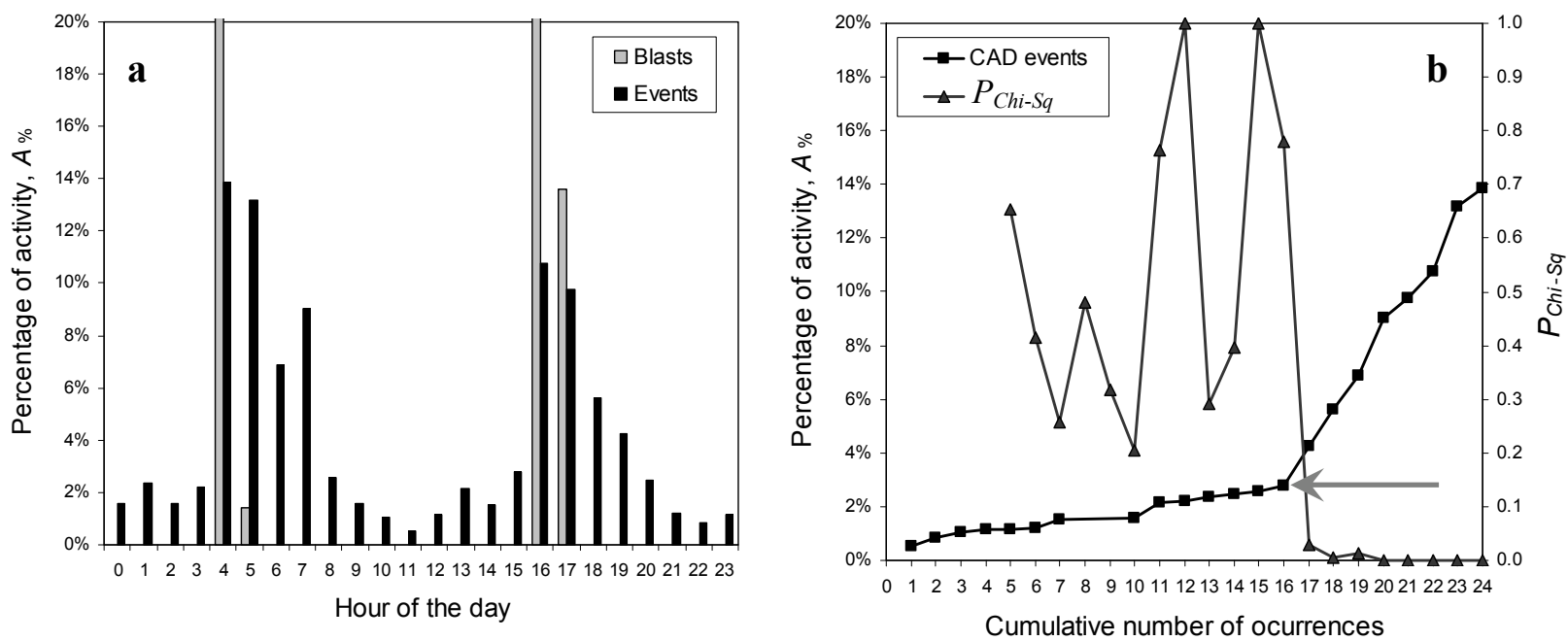

Figure 7 (a) Event rate diurnal chart for a zone of a mine; (b) cumulative ascending distribution of the hourly activity and observed level of significance of the Chi-Square test $\left(P_{C h i}{ }^{-S q}\right)$ for testing the hypothesis that the data set can be described by a uniform distribution

In order to reduce the subjectivity of the selection of the hours of highest activity and automate the procedure the cumulative ascending distribution (CAD) for the sample data of $A_{\%}$ (squares in Figure $7 \mathrm{~b}$ ) is examined. 
The result indicates that there is a change point in the population that divides the hours of low and high activity (horizontal arrow in Figure $7 \mathrm{~b})$. The observed level of significance of the Chi-Square test $\left(P_{\text {Chi-Sq }}\right)$ (triangles in Figure 7b) is used to test the hypothesis that the data set can be described by a uniform distribution for some $A_{\%}$. The change point is determined at the first $A_{\%}$ where the data is considered to follow a uniform distribution with a significance level of 0.2 . This point is also coincident with the previously visually determined horizontal arrow in Figure $7 \mathrm{~b}$.

\subsection{Application examples}

Most of the sequences analysed presented a type 1 pattern (Figure 6). Two particular and interesting type 2 paths are presented to illustrate the concepts and the application of the proposed methodology (Figure 8). In both sequences the event rate is continuously evaluated using a time bin of two hours with a 0.1 hour shift. The maximum curvature boundary is used as the minimum time to evaluate the behaviour of the seismic sequence after the initial event. In addition, two and three times the background level $B$ is used as observation time windows to evaluate the irregularity or stabilisation in the decay of aftershock events before reaching $B$. The crosses of the seismic path with the corresponding background level are indicated by squares in the examples. Furthermore, a magnitude-time plot is included as a reference.

The first example (Figure 8I) corresponds to a rockburst $\left(M_{n}=3.8\right)$. This sequence was accompanied by a $\mathrm{M}_{\mathrm{n}}=1.5$ and $\mathrm{a} \mathrm{M}_{\mathrm{n}}=1.1,0.3$ and 3.4 hours after the first event. The data crosses several envelopes $(45 \%$ to $75 \%$ in Figure 8Ia) in a sub-vertical direction before the $M_{n}=1.1$ indicating from the very beginning a delayed ground response. After the $\mathrm{Mn}=1.1$, seismicity builds up until touching the $85 \%$ envelope, where the maximum curvature boundary is crossed at hour 6.2 (Figure $8 \mathrm{Ib}$ ). After the maximum curvature boundary is crossed the event rate decays in only 0.2 and 0.7 hours to $3 B$ and $2 B$ respectively. Given the short time delay between $T_{M C}$ and $3 B, 2 B$ it is necessary to further wait until the event rate decays to $B$. A small stabilisation is observed in the event rate (Figure $8 \mathrm{Ib}$ ) after $2 B$ is observed, until it decays to $B$ at hour 9.2. At that time the seismic path (Figure 8Ia) has started to cross the $85 \%$ seismic envelope in a subhorizontal direction. This is the re-entry time. Note that additional seismicity occurs after $B$ but they all correspond to small magnitude events (Figure 8Ic).

The second example (Figure 8II) presents a large magnitude event $\left(\mathrm{M}_{\mathrm{n}}=2.0\right)$ with no initial damage. The maximum curvature boundary, $3 B$ and $2 B$ background levels are crossed 5.4, 5.2 and 5.7 hours after the $\mathrm{M}_{\mathrm{n}}=2.0$ respectively (Figure $8 \mathrm{IIb}$ ). At this time the seismic path has already crossed in a sub-vertical direction the 65 to $75 \%$ envelopes (Figure 8IIa) indicating a delayed ground response. Given the irregularity of the event rate and seismic path in a sub-vertical direction it is necessary to wait to further evaluate the seismic response. The event rate locally reaches the $B$ background level 16.2 hours after the main event (Figure 8IIb). Until that time the seismic path continued crossing seismic envelopes in a sub-vertical direction, which is reflected by oscillations on the event rate between $2 B$ and $B$. Additional seismicity enters at approximately $06 / 21 / 2007$ : 18 . This creates a spike in seismicity rate that continues until 06/22/2007: 0 , where the seismicity rate locally returns to $B$ (Figure $8 \mathrm{IIb}$ ). This spike is also associated with an increase in the magnitude of the events (Figure 8IIc). After the spike an oscillation of the rate is observed between $B$ and $2 B$ before the $\mathrm{M}_{\mathrm{n}}=0.5$ event. During this time period the seismic path crosses an additional seismic envelope (87.5\%) in a sub-vertical direction. After the $\mathrm{M}_{\mathrm{n}}=0.5$ event the seismic path and rate shows a steady increase that leads to the $\mathrm{M}_{\mathrm{n}}=2.2$ rockburst. Although the formal re-entry time is 16.2 hours, i.e., once $B$ is reached for the first time, the low speed of decay compared to the average of the site, and the additional seismicity measured from 06/21/2007: 18 to 06/22/2007: 0, may suggest a continuation of the restriction until the decay starts crossing the seismic envelopes in a sub-horizontal direction and the event rate stabilises around $B$. 


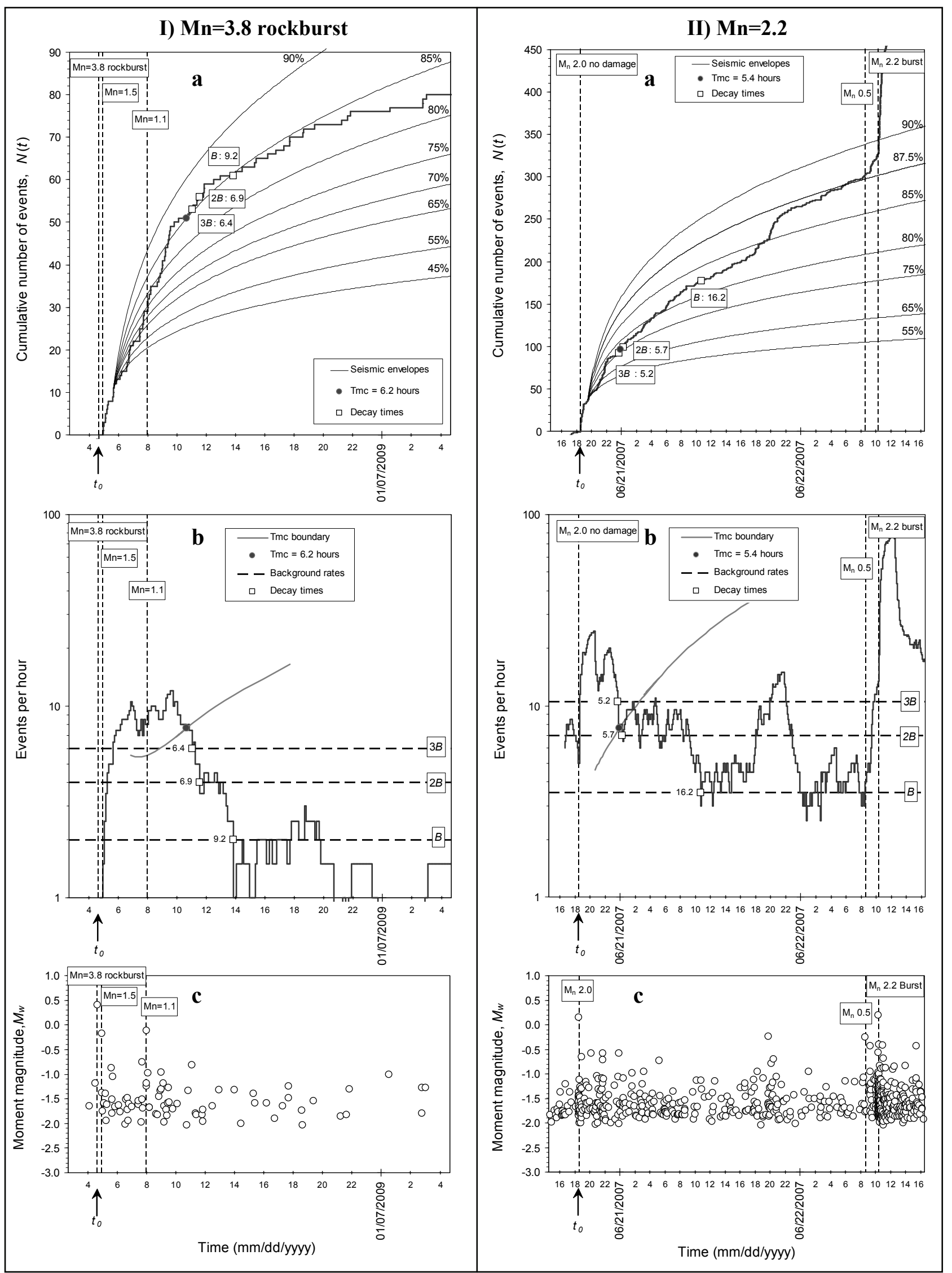

Figure 8 Application examples presenting a type 2 pattern: (a) seismic envelopes; (b) event rate; (c) magnitude-time plot. Large magnitude events are displayed by vertical dashed lines 


\section{Conclusions}

Aftershock time sequences are complex in nature, nevertheless different seismic environments presented common statistical properties that are valuable in terms of application for re-entry protocol development. The statistics are site specific however the methodology is widely applicable.

To provide insight into the decay patterns of an on-going sequence the concept of seismic envelopes was developed. With this family of decay-law curves it is possible to evaluate the path and decay pattern of a new large magnitude event, rockburst or blast. The approach considered here is to analyse several mining seismic sequences in order to identify the natural response of the rock mass to the mining process.

Considering the possible explanation of $p$ values higher than 1 offered by Dieterich (1994): the shear stress applied to the fault after the main shock may decrease with time, and the experimental results of Lockner (1993), and Rudajev et al. (1996): the $p$ value decreases with increasing load level. Therefore, sequences with speed of decays lower than the average and seismicity rate higher than backgrounds can be interpreted as cases where stresses are increasing with time following the main event. The prediction of potentially damaging events is outside of the scope of a re-entry protocol, however, as a preventative measure re-entry should be delayed until the decay starts crossing the seismic envelopes in a sub-horizontal direction and the rate approaches the background level of seismicity. It is worthwhile to examine more field evidence to test this concept.

By evaluating the seismic path in-between observation time windows for different levels of background it is possible to interpret in a better manner the decay patter of the seismic sequence and manage more effectively the risk after large magnitude events/rockbursts or blasts.

The proposed methodology has been extended to account for magnitude in the re-entry protocol by using seismic work instead of event rate (Vallejos, 2010) and can be applied to any seismic source parameter of interest for which it is possible to fit a functional form of the power-law type.

\section{Acknowledgements}

This project was identified and supported by the MASHA Ground Control Committee (Mines and Aggregates Safety and Health Association) and funded by WSIB (Workplace Safety and Insurance Board). The authors wish to acknowledge the permission of the mines to use their data and publish this work.

\section{References}

Anderson, T.W. and Darling, D.A. (1954) A test of goodness of fit, Journal of the American Statistical Association, Vol. 49, pp. 765-769.

Das, S. and Scholz, C.H. (1981) Theory of time-dependent rupture in the Earth, Journal of Geophysical Research, Vol. 86, pp. 6039-6051.

Davis, S.D. and Frohlich, C. (1991a) Single-link cluster analysis, synthetic earthquake catalogues, and aftershock identification, Geophysical Journal International, Vol. 104, pp. 289-306.

Davis, S.D. and Frohlich, C. (1991b) Single-link cluster analysis of earthquake aftershocks: decay laws and regional variations, Journal of Geophysical Research, Vol. 96, pp. 6335-6350.

Dieterich, J.A. (1994) A constitutive law for rate of earthquake production and its application to earthquake clustering, Journal of Geophysical Research, Vol. 99, pp. 2601-2618.

Frohlich, C. and Davis, S. (1985) Identification of aftershocks of deep earthquakes by a new ratios method, Geophysical Research Letters, Vol. 12, No. 10, pp. 713-716.

Gasperini, P. and Lolli, B. (2006) Correlation between the parameters of the rate equation for simple aftershock sequences: implications for the forecasting of rates and probabilities, Physics of the Earth and Planetary Interiors, Vol. 156, No. 1-2, pp. 41-58.

Gross, S.J. and Kisslinger, C. (1994) Test of models of aftershock rate decay, Bulleting of the Seismological Society of America, Vol. 84, pp. 1571-1579.

Heal, D., Hudyma, M. and Vezina, F. (2005) Seismic hazard at Agnico-Eagle's Laronde Mine using MS-RAP, Maintenance Engineering and Mine Operators Conference, Sudbury, Canada.

Hudyma, M., Heal, D. and Mikula, P. (2003) Seismic monitoring in mines-Old technology, new applications, in Proceedings 1st Australian Ground Control in Mining Conference: Technology \& Practice, Sydney, Australia, pp. 201-218. 
Isacks, B.L., Sykes, L.R. and Oliver, J. (1967) Spatial and temporal clustering of deep and shallow earthquakes in the Fiji-Tonga-Kermadec region, Bulletin of the Seismological Society of America, Vol. 57, pp. 935-958.

Kagan, Y.Y. and Knopoff, L. (1976) Statistical search for non-random features of the seismicity of strong earthquakes, Physics of the Earth and Planetary Interiors, Vol. 12, pp. 291-318.

Kagan, Y.Y. (2004) Short-term properties of earthquakes catalogs and models of earthquake source, Bulletin of the Seismological Society of America, Vol. 94, No. 4, pp. 1207-1228.

Kagan, Y.Y. and Jackson, D.D. (2006) Comment on Testing earthquake prediction methods: "The West Pacific shortterm forecast of earthquakes with magnitude MwHRV $\geq 5.8$ " by V.G. Kossobokov, Tectonophysics, Vol. 413, pp. 33-38.

Kisslinger, C. and Jones, L.M. (1991) Properties of aftershock sequences in Southern California, Journal of Geophysical Research, Vol. 96(B7), pp. 11,947-11,958.

Kisslinger, C. (1996) Aftershocks and fault zone properties, Advances in Geophysics, Vol. 38, pp. 1-36.

Lockner, D. (1993) The role of acoustic emission in the study of rock fracture, International Journal of Rock Mechanics and Mining Science \& Geomechanics Abstracts, Vol. 30, No. 7, pp. 883-899.

Mogi, K. (1967) Earthquakes and fractures, Tectonophysics, Vol. 5, pp. 35-55.

Malek, F. and Leslie, I.S. (2006) Using seismic data for rockburst re-entry protocol at Inco's Copper Cliff North Mine, in Proceedings 41st US Rock Mechanics Symposium, ARMA/USRMS, June 2006, Golden Colorado, paper 061163.

Molchan, G.M. and Dmitrieva, O.E. (1992) Aftershock identification: methods and new approaches, Geophysical Journal International, Vol. 109, pp. 501-516.

Nyffenegger, P. (1998) Aftershock occurrence rate decay for individual sequences and catalogs, PhD thesis, University of Texas at Austin, $131 \mathrm{p}$.

Nyffenegger, P. and Frohlich, C. (1998) Recommendations for determining p values for aftershock sequences and catalogs, Bulletin of the Seismological Society of America, Vol. 88, pp. 1144-1154.

Nyffenegger, P. and Frohlich, C. (2000) Aftershock occurrence rate decay properties for intermediate and deep earthquake sequences, Geophysical Research Letters, Vol. 27, pp. 1215-1218.

Ogata, Y. (1983) Estimation of the parameters in the modified Omori formula for aftershock frequencies by the maximum likelihood procedure, Journal of Physics of the Earth, Vol. 31, pp. 115-124.

Ogata, Y. (1988) Statistical models for earthquake occurrences and residual analysis for point processes, Journal of the American Statistical Association, Vol. 83, pp. 9-27.

Omori, F. (1894) On the after-shocks of earthquakes, The Journal of the College of Science, Imperial University of Tokyo, Vol. 7, pp. 111-200.

Potvin, Y. (2008) Strategies and tactics to control seismic risks in mines, in Proceedings 3rd International Seminar on Strategic vs Tactical Approaches in Mining, Québec, Canada, pp. 195-209.

Reasenberg, P. (1985) Second-order moment of central California seismicity, 1969-1982, Journal of Geophysical Research, Vol. 90, pp. 5479-5495.

Reasenberg, P. and Jones, L.M. (1989) Earthquake hazard after a mainshock in California, Science, Vol. 243, pp. 1173-1176.

Rudajev, V., Vilhelm, J., Kozak, J. and Lokajicek, T. (1996) Statistical precursors of instability of loaded rock samples based on acoustic emission, International Journal of Rock Mechanics and Mining Science \& Geomechanics Abstracts, Vol. 33, No. 7, pp. 743-748.

SeisWatch (2007) ESG Hyperion Software User's Guide-v13.0, Vol. 2, Chapter 4.

Scholz, C.H. (2003) The mechanics of earthquakes and faults, Second edition, Cambridge University Press, Cambridge, $471 \mathrm{p}$.

Shcherbakov, R. and Turcotte, D.L. (2004) A damage mechanics model for aftershocks, Pure and Applied Geophysics, Vol. 161, pp. 2379-2391.

Shlien, S. and Toksoz, M.N. (1974) A statistical method of identifying dependent events and earthquake aftershocks, Earthquake Notes, Vol. 45, pp. 3-16.

Utsu, T. (1961) A statistical study of the occurrence of aftershocks, Geophysical Magazine, Vol. 30, pp. $521-605$.

Utsu, T., Ogata, Y. and Matsu'ura, R. (1995) The centenary of the Omori formula for a decay law of aftershock activity, Journal of Physics of the Earth, Vol. 43, No. 1, pp. 1-33.

Vallejos, J.A. and McKinnon, S.D. (2008) Guidelines for development of re-entry protocols in seismically active mines, in Proceedings 42nd US Rock mechanics symposium, ARMA/USRMS, June 2008, San Francisco, California, paper 08-97.

Vallejos, J.A. and McKinnon, S.D. (2009) Re-entry protocols for seismically active mines using statistical analysis of aftershock sequences, in Proceedings 20th Canadian Rock Mechanics Symposium, May 2009, Toronto, Canada, paper 4028 .

Vallejos, J.A. and McKinnon, S.D. (2010) Omori's law applied to mining-induced seismicity and re-entry protocol development, Pure and Applied Geophysics, Vol. 167, No. 1, pp. 91-106. 
Vallejos, J.A. (2010) Analysis of seismicity in mines and development of re-entry protocols, $\mathrm{PhD}$ thesis, Queen's University, Kingston, Canada, 403 p.

Van Dusen, J. and Shumila, V. (2001) ESG-Internal presentation to Creighton Mine on enhancing rockburst re-entry procedures using seismic source parameters (SeisWatch). 